\title{
Microbiota intestinal y obesidad
}

\author{
Yoel López Gamboa \\ yoel111975@gmail.com \\ https://orcid.org/0000-0002-9596-443X \\ Yoeldis Gamboa Pellicier, \\ gamboa41@gmail.com \\ Yanet Rodríguez Cantillo \\ alfrelia@gmail.com \\ https://orcid.org/0000-0001-5028-9576
}

Universidad Metropolitana de Ecuador

Ciudad de Guayaquil. Provincia Guayas. Ecuador

\section{RESUMEN}

La obesidad está definida como un aumento de la grasa corporal en proporción a la altura del paciente. El diagnóstico se establece con la medición del Índice de Masa Corporal (IMC); el mismo se calcula dividiendo el peso en kilogramos entre la altura en metros al cuadrado. Investigaciones actuales han evidenciado que existe relación entre el microbiota intestinal y la obesidad. El objetivo del presente trabajo estuvo dirigido a describir tal asociación. Se realizó una investigación documental mediante revisión bibliográfica en las bases de datos Google académico, SciELO, Medline, Dialnet, y PubMed; con las palabras claves: obesidad, inflamación, microbioma intestinal y probióticos. La revisión se realizó en los idiomas inglés, español y portugués. Se utilizó bibliografía de los últimos 5 años. Se ha identificado a través de diferentes estudios que en las personas con exceso de peso existen modificaciones en la composición de su flora intestinal, disminuyendo considerablemente las cantidades de bifidobacterias y lactobacilos, a la vez que se incrementan otros gérmenes fundamentalmente bacterias gran negativas que tienen en sus membranas como componente mayoritario lipopolisacáridos que puede estimular la inflamación. A estos gérmenes también se les atribuye la propiedad de hacer más rentable la energía de los alimentos, por lo que son capaces de obtener más energía de la misma ingesta calórica diaria. Las investigaciones actuales muestran que existe relación entre la obesidad y la característica del microbiota intestinal, destacando que en los pacientes obesos existe un incremento de bacteroidetes y menor proporción de gérmenes firmicutes.

Palabras claves: obesidad; inflamación; microbioma intestinal; probióticos 


\title{
Gut Microbiota and obesity
}

\begin{abstract}
Obesity is defined as an increase in body fat in proportion to the height of the patient. The diagnosis is established with the measurement of the Body Mass Index (BMI); It is calculated by dividing the weight in kilograms by the height in meters squared. Current research has shown that there is a relationship between the intestinal microbiota and obesity. The objective of the present work was aimed at describing such an association. A documentary research was carried out by means of a bibliographic review in the Google academic, SciELO, Medline, Dialnet, and PubMed databases; with the keywords: obesity, inflammation, gut microbiome and probiotics. The review was carried out in the English, Spanish and Portuguese languages. Bibliography of the last 5 years was used. It has been identified through different studies that in people with excess weight there are modifications in the composition of their intestinal flora, considerably reducing the amounts of bifidobacteria and lactobacilli, at the same time that other germs are increased, fundamentally great negative bacteria that they have in its membranes as a major component lipopolysaccharides that can stimulate inflammation. These germs are also attributed the property of making the energy from food more profitable, so they are able to obtain more energy from the same daily caloric intake. Current research shows that there is a relationship between obesity and the characteristic of the intestinal microbiota, highlighting that in obese patients there is an increase in bacteroidetes and a lower proportion of firmicutes germs.
\end{abstract}

Keywords: obesity; inflammation; gut microbiome; probiotics

Artículo recibido: 05 octubre. 2021 Aceptado para publicación: 02 noviembre 2021

Correspondencia: yoel111975@gmail.com Conflictos de Interés: Ninguna que declarar 


\section{INTRODUCCIÓN}

El concepto de obesidad está definido como un aumento de la grasa corporal en proporción a la altura del paciente. El diagnóstico se establece con la medición del Índice de Masa Corporal (IMC); el cual se calcula dividiendo el peso en kilogramos entre la altura en metros al cuadrado. Un IMC entre 18, 6 y 24,9 es considerado normal y entre 25 y $29,9 \mathrm{~kg} / \mathrm{m}^{2}$ determina el diagnóstico de sobrepeso, mientras que un valor mayor o igual a $30 \mathrm{~kg} / \mathrm{m}^{2}$ se considera como obesidad. Valores por encima de 40 en el IMC expresan obesidad mórbida. Tanto la prevalencia del sobrepeso como de la obesidad han aumentado considerablemente en los últimos 10 años tanto en nuestro país como a nivel mundial, y en algunos países como EE. UU es considerado una epidemia. El peso corporal de una persona está determinado por la relación de varios factores, dentro de los cuales se encuentran: factores ambientales, culturales, genéticos, sociales y el gasto energético, donde juega un papel determinante el nivel de actividad física de los individuos. (Pothuraju \& Sharma, 2018)

Investigaciones de los últimos años han evidenciado que existe relación entre el microbiota intestinal y el desarrollo de diferentes enfermedades, entre ellas diabetes y la obesidad. Esta asociación se ha establecido por la identificación de diferencias entre los gérmenes que colonizan el intestino de las personas obesas y las personas que tienen un peso normal. Los investigadores han atribuido la asociación entre el microbiota intestinal y la obesidad a que la alteración en el microbiota repercute en la extracción energética de los alimentos, el metabolismo de los ácidos grasos, la síntesis de hormonas intestinales que intervienen en el equilibrio energético, y la regulación de los depósitos corporales del tejido adiposo. (Abenavoli et al., 2019), («La importancia de la microbiota en la obesidad», 2017)

Durante los últimos diez años mediante el estudio del ARN ribosomal de las bacterias ha permitido conocer la composición de la flora intestinal, e identificar más de 400 especies de bacterias diferentes en el colon de los seres humanos, y dentro de ellas más de 200 géneros, siendo los más significativos micoplasmas, bacillus y clostridium. (Pothuraju \& Sharma, 2018),(Jedwab et al., 2021)

Existen varios factores que intervienen en la composición de la flora del intestino del organismo humano, dentro de ellos el tipo de parto y la lactancia materna juegan un papel preponderante en el primer año de vida, después de ese período se conjugan otras 
variables como la dieta, la genética y factores ambientales dentro de los que se incluye el consumo de antibióticos. (de Clercq et al., 2016), (Guillot, s. f.)

La cantidad de microorganismos que viven en contacto con el hombre es muy superior al número de células que conforman el organismo humano. Solamente en el intestino grueso de los mamíferos el número de microorganismos que existen se ubica entre 10 a la 12 y 10 a la 14 lo que equivale a aproximadamente entre $1-1.5 \mathrm{~kg}$ de peso, que en ocasiones puede ser superior a las cantidades encontradas en el suelo. (Torres-Fuentes et al., 2017).Con el objetivo de describir la relación que existe entre la obesidad y la composición del microbioma intestinal se realiza la presente investigación.

\section{MÉTODOS}

Se realizó una investigación documental mediante revisión bibliográfica del estado actual del tema en las bases de datos Google académico, SciELO, Medline, Dialnet, y PubMed; con las palabras claves: obesidad, inflamación, microbioma intestinal y probióticos; buscando algún nivel de relación entre la obesidad y el microbioma intestinal. La revisión se realizó en los idiomas inglés español y portugués. Se utilizó bibliografía de los últimos 5 años. No obstante, se tuvieron en cuenta publicaciones precedentes con elementos relevantes que pudieran explicar el objetivo propuesto. Se encontraron 68 artículos con temáticas relacionadas, de los cuales 29 fueron tomados en consideración por abordar con mayor precisión el tema en cuestión.

\section{RESULTADOS Y DISCUSIÓN}

La flora intestinal de cada individuo es única, y se forma desde el momento del parto, y generalmente se mantiene en constante cambios hasta el primer año de vida. Existen diferentes factores desde el nacimiento que condicionan la composición de los gérmenes que se desarrollan en el sistema digestivo. Se ha demostrado que la flora intestinal de los niños que nacen por parto natural, los gérmenes que habitan su sistema digestivo coinciden con los existente en la vagina de la madre, sin embargo, los nacidos por cesárea desarrollan con mayor frecuencia los gérmenes que les trasmite el personal sanitario que realiza los procederes del parto. En la mayoría de los individuos los gérmenes que colonizan su sistema digestivo se corresponden con bifidobacterias y lactobacilos. (Halmos \& Suba, 2016), (González-Gallegos et al., 2017)

Se ha identificado a través de diferentes estudios que en las personas con exceso de peso existen modificaciones en la composición de su flora intestinal, disminuyendo 
considerablemente las cantidades de bifidobacterias y lactobacilos, a la vez que se incrementan otros gérmenes fundamentalmente bacterias gran negativas que tienen en sus membranas como componente mayoritario lipopolisacáridos que puede estimular la inflamación. A estos gérmenes también se les atribuye la propiedad de hacer más rentable la energía de los alimentos, por lo que son capaces de obtener más energía de la misma ingesta calórica diaria. (Cuevas-Sierra et al., 2019)

La flora intestinal expresa una gran variedad de enzimas glicósido hidrolasas que no están presentes en el genoma de los humanos, las cuales tienen como función la trasformación de los polisacáridos complejos de la dieta en monosacáridos y ácidos grasos de cadena corta, entre los que se encuentran butirato, acetato y propionato fundamentalmente. Entre los ácidos grasos de cadena corta y los monosacáridos constituyen una fuente importante de energía para los individuos ya que se estima un aporte energético aproximadamente del 10 por ciento de la energía que el organismo absorbe. Las evidencias apuntan a que el microbiota de las personas con exceso de peso tiene mayor capacidad de degradar los carbohidratos no digeribles de los vegetales y por consiguiente favorecen la absorción de energía y con ello el exceso de peso. (Hills et al., 2019)

Con la modificación de la flora intestinal en los pacientes con exceso de peso también existen otras asocianes relacionadas con el estreñimiento, el incremento de la probabilidad de cáncer de colon, Alzheimer, ansiedad y depresión. (Jiang et al., 2017), (Minayo et al., 2021). Y en el caso de los niños se pueden manifestar enfermedades alérgicas, hígado graso no alcohólico, enfermedades inflamatorias a nivel intestinal y también obesidad. (Yang \& Ni, 2019)

En las personas que tienen sobrepeso u obesidad existen cambios sustanciales en su microbiota intestinal demostrándose que la mayoría de los pacientes presentan una mayor cantidad de bacterias gramnegativas (bacteroidetes) y menor proporción de gérmenes grampositivos (firmicutes). Dentro de los gérmenes que pueden ser potencialmente dañino en las personas con sobrepeso y obesidad se encuentran entre otros, clostridios, staphylococcus aureus o ciertos bacteroides. (Fontané et al., 2018b)

Diferentes estudios en animales han demostrado algunos de los cambios que pudieran explicar la influencia de la flora intestinal en la obesidad, ya que existen hallazgos que expresan que la flora en obesos se relaciona con la expresión genética de algunas 
enzimas que participan en la extracción de nutrientes, mayor fermentación a nivel intestinal y disminución de las calorías residuales en las deposiciones. También se ha logrado demostrar que al trasplantar deposiciones de animales obesos a animales normopeso, los normopeso desarrollaban obesidad en periodos muy cortos de días. (Pushpanathan et al., 2019)

Un elemento a considerar como de extrema importancia en el mantenimiento del microbioma intestinal es la calidad del sueño, que actualmente se conoce que también puede impactar a los gérmenes que colonizan nuestro sistema digestivo que siguen ritmos circadianos, manteniendo un nivel de actividad mayor durante el día, mientras en el horario nocturno se ha evidenciado un nivel de actividad menor, que al final se traduce en un ineficiente funcionamiento del metabolismo. (Matenchuk et al., 2020)

El uso de productos probiótico y prebióticos han mostrado resultados positivos en la modificación de la composición de la flora intestinal y consiguientemente en el tratamiento del exceso de peso. Los prebióticos al ser sustancias que el organismo no digiere con facilidad deben ser degradado por fermentación a través de la acción de los gérmenes que habitan el sistema digestivo, lo que favorece el crecimiento y desarrollo de la flora intestinal (aumenta las cantidades de lactobacilos y bifidobacterias a la vez que reduce el crecimiento de otras especies de bacterias). Dentro de los prebióticos más usados a nivel industrial en la actualidad se encuentran la inulina y los fructoologosacáridos. (Sergeev et al., 2020)

Los probiótico al ser microorganismos vivos, en su mayoría bacterias productoras de ácido láctico (bifidobacterias y lactobacilos) aportan varios beneficios en el control de peso corporal. Los mecanismos de acción de los probiótico se han definido varios, los que cursan fundamentalmente modificando la flora intestinal debido a la liberación de sustancias antimicrobiana tales como; defensinas, bacteriocinas, peróxido de hidrógenos, entre otras que provocan la inhibición del crecimiento de otros gérmenes patógenos, y de gérmenes más eficientes en la obtención energética de los alimentos, también favorecen la producción de ácidos grasos de cadena corta, e intervienen en la modificación del pH del tracto intestinal. Los probióticos compuestos por lactobacilos y bifidobacterias que constituyen la mayoría de los gérmenes que colonizan el sistema digestivo humano logran equilibrar la fermentación de los sustratos no absorbibles o poco absorbibles y con ello el metabolismo energético que los mismo aportan y que 
influyen en la composición corporal. Existen varios productos que en la actualidad mezclan prebióticos y probióticos para lograr efectos sinérgicos en el control del peso corporal, y precisamente éstas mezclas denominadas simbióticos son los que han evidenciado mejores resultados. (Sergeev et al., 2020),

El efecto del microbiota intestinal cada vez cobra mayor relevancia en el mantenimiento del estado de salud, pues impacta mucho más de lo que se creía; recientes investigaciones han atribuidos a las bacterias que habitan el sistema digestivo de los obesos responsabilidad en el desarrollo de osteoartrosis, proceso inflamatorio que guarda relación con el sistema inmunológico innato. (Liu et al., 2019, p.). Se les atribuye a los probióticos (lactobacilos y bifidobacterias) también propiedades reguladoras sobre los triglicéridos, el colesterol, la glucosa en sangre y sobre la liberación de insulina al sistema circulatorio. (Hu et al., 2017), (Fontané et al., 2018a)

Los probiótico como se ha explicado desempeñan un papel significativo en el metabolismo intestinal y el sistema inmune, por lo que son considerado como una alternativa importante para restablecer condiciones de disbiosis de la flora intestinal que guarda relación con el sobre peso y la obesidad. De manera similar el prebiótico favorece la fermentación que involucra el microbiota intestinal y con ello el desarrollo de gérmenes implicados en el equilibrio de la flora intestinal. También se obtienen metabolitos de las bacterias, especialmente los ácidos grasos de cadena corta los que pueden actuar directa o indirectamente en las células intestinales (mediante la modificación del $\mathrm{pH}$ ), regulando varios procesos como la carcinogénesis colorrectal, la absorción de minerales, y la eliminación de otras sustancias dañina al organismo humano. (Reyes-Muñoz, Sosa, Flores-Robles, Arce-Sánchez, Martínez-Cruz, \& Gutiérrez-Castrellón, 2020), (Reyes-Muñoz, Sosa, Flores-Robles, Arce-Sánchez, Martínez-Cruz, Garduño-García, et al., 2020)

Otras investigaciones apuntan también a los efectos beneficiosos de los prebióticos en el control del metabolismo de lípidos, y disminución de riesgos en las enfermedades cardiovascular, dichos efectos se atribuyen fundamentalmente a que los prebióticos favorecen la fermentación de algunos lactobacilos a los que se les ha atribuido la mencionada propiedad, mientras que las investigaciones no han mostrado asociación entre el metabolismo de lípidos y las bifidobacterias. (Vizcaíno et al., 2016), (Castro et al., 2016) 


\section{CONCLUSIONES}

Las investigaciones actuales muestran que existe relación entre la obesidad y la característica del microbiota intestinal, destacando que en los pacientes obesos existe un incremento de bacteroidetes y menor proporción de gérmenes firmicutes; conociéndose que los bacteroidetes son mucho más eficiente en la extracción de energía de los alimentos. Se necesitan más estudios para definir si es causa o consecuencia la disbiosis intestinal en el caso de la obesidad.

\section{REFERENCIAS BIBLIOGRÁFICAS}

Abenavoli, L., Scarpellini, E., Colica, C., Boccuto, L., Salehi, B., Sharifi-Rad, J., Aiello, V., Romano, B., De Lorenzo, A., Izzo, A. A., \& Capasso, R. (2019). Gut Microbiota and Obesity: A Role for Probiotics. Nutrients, 11(11), E2690. https://doi.org/10.3390/nu11112690

Castro, M., Arias, I., Barboza, F., Duque, D. L., \& Villalobos, D. (2016). Usos clínicos de los probióticos: Malabsorción de lactosa, cólico del lactante, enfermedad inflamatoria intestinal, enterocolitis necrotizante, Helycobacter pylori. Archivos Venezolanos de Puericultura y Pediatría, 79(1), 022-028.

Cuevas-Sierra, A., Ramos-Lopez, O., Riezu-Boj, J. I., Milagro, F. I., \& Martinez, J. A. (2019). Diet, Gut Microbiota, and Obesity: Links with Host Genetics and Epigenetics and Potential Applications. Advances in Nutrition (Bethesda, Md.), 10(suppl_1),S17-S30. https://doi.org/10.1093/advances/nmy078

de Clercq, N. C., Groen, A. K., Romijn, J. A., \& Nieuwdorp, M. (2016). Gut Microbiota in Obesity and Undernutrition. Advances in Nutrition (Bethesda, Md.), 7(6), 1080-1089. https://doi.org/10.3945/an.116.012914

Fontané, L., Benaiges, D., Goday, A., Llauradó, G., \& Pedro-Botet, J. (2018a). Influencia de la microbiota y de los probióticos en la obesidad. Clínica e Investigación en Arteriosclerosis, 30(6), 271-279. https://doi.org/10.1016/j.arteri.2018.03.004

Fontané, L., Benaiges, D., Goday, A., Llauradó, G., \& Pedro-Botet, J. (2018b). Influence of the microbiota and probiotics in obesity. Clinica E Investigacion En Arteriosclerosis: Publicacion Oficial De La Sociedad Espanola De Arteriosclerosis, 30(6), 271-279. https://doi.org/10.1016/j.arteri.2018.03.004 
González-Gallegos, N., González-Torres, Y. S., \& Padilla-Durán, L. F. (2017). Microbiota intestinal, sobrepeso y obesidad. Revista Salud Pública y Nutrición, 16(3), 23-28.

Guillot, C. C. (s. f.). Microbiota intestinal y obesidad en la infancia. Revista Cubana de Pediatría., 24.

Halmos, T., \& Suba, I. (2016). [Physiological patterns of intestinal microbiota. The role of dysbacteriosis in obesity, insulin resistance, diabetes and metabolic $\begin{array}{llll}\text { syndrome]. } & \text { Orvosi } & \text { Hetilap, } & \text { 157(1), }\end{array}$ https://doi.org/10.1556/650.2015.30296

Hills, R. D., Pontefract, B. A., Mishcon, H. R., Black, C. A., Sutton, S. C., \& Theberge, C. R. (2019). Gut Microbiome: Profound Implications for Diet and Disease. Nutrients, 11(7), E1613. https://doi.org/10.3390/nu11071613

Hu, Y.-M., Zhou, F., Yuan, Y., \& Xu, Y.-C. (2017). Effects of probiotics supplement in patients with type 2 diabetes mellitus: A meta-analysis of randomized trials. Medicina Clinica, 148(8), 362-370. https://doi.org/10.1016/j.medcli.2016.11.036 Jedwab, C. F., Roston, B. C. de M. B., Toge, A. B. F. de S., Echeverria, I. F., Tavares, G. O. G., Alvares, M. A., Rullo, V. E. V., \& Oliveira, M. R. M. de. (2021). The role of probiotics in the immune response and intestinal microbiota of children with celiac disease: A systematic review. Revista Paulista de Pediatria, 40. https://doi.org/10.1590/1984-0462/2022/40/2020447

Jiang, C., Li, G., Huang, P., Liu, Z., \& Zhao, B. (2017). The Gut Microbiota and Alzheimer's Disease. Journal of Alzheimer's Disease: JAD, 58(1), 1-15. https://doi.org/10.3233/JAD-161141

La importancia de la microbiota en la obesidad. (2017). Revista Española $\begin{array}{llll}\text { Endocrinología } & \text { Pediátrica, } & 8 & \text { Suppl. }\end{array}$ https://doi.org/10.3266/RevEspEndocrinolPediatr.pre2017.Apr.394

Liu, Y., Ding, W., Wang, H. L., Dai, L. L., Zong, W. H., Wang, Y. Z., Bi, J., Han, W., \& Dong, G. J. (2019). Gut microbiota and obesity-associated osteoarthritis. $\begin{array}{llll}\text { Osteoarthritis } \quad \text { and } \quad \text { Cartilage, 27(9), } & \text { 1257-1265. }\end{array}$ https://doi.org/10.1016/j.joca.2019.05.009 
Matenchuk, B. A., Mandhane, P. J., \& Kozyrskyj, A. L. (2020). Sleep, circadian rhythm, and gut microbiota. Sleep Medicine Reviews, 53, 101340. https://doi.org/10.1016/j.smrv.2020.101340

Minayo, M. de S., Miranda, I., \& Telhado, R. S. (2021). Revisão sistemática sobre os efeitos dos probióticos na depressão e ansiedade: Terapêutica alternativa? Ciência \& Saúde Coletiva, 26, 4087-4099. https://doi.org/10.1590/141381232021269.21342020

Pothuraju, R., \& Sharma, R. K. (2018). Interplay of Gut Microbiota, Probiotics in Obesity: A Review. Endocrine, Metabolic \& Immune Disorders Drug Targets, 18(3), 212-220. https://doi.org/10.2174/1871530318666180131092203

Pushpanathan, P., Mathew, G. S., Selvarajan, S., Seshadri, K. G., \& Srikanth, P. (2019). Gut microbiota and its mysteries. Indian Journal of Medical Microbiology, 37(2), 268-277. https://doi.org/10.4103/ijmm.IJMM_19_373

Reyes-Muñoz, E., Sosa, S. E. Y., Flores-Robles, C. M., Arce-Sánchez, L., MartínezCruz, N., Garduño-García, G., Tawney-Serrano, C. R., Domínguez-Rodríguez, J. J., Martínez-Hernández, M. L., Pérez-Mota, L. R., Llanes-Carrillo, L. C., \& González-Rodríguez, M. (2020). Uso de mioinositol más Bifidobacterium lactis y Lactobacillus rhamnosus para la prevención de diabetes mellitus gestacional en mujeres mexicanas. Gaceta Medica De Mexico, 156(Supl 3), S51-S57. https://doi.org/10.24875/GMM.M20000438

Reyes-Muñoz, E., Sosa, S. E. Y., Flores-Robles, C. M., Arce-Sánchez, L., MartínezCruz, N., \& Gutiérrez-Castrellón, P. (2020). Suplementos nutricionales para prevención de diabetes mellitus gestacional: Lecciones aprendidas basadas en la evidencia. Gaceta Medica De Mexico, 156(Supl 3), S43-S50. https://doi.org/10.24875/GMM.M20000437

Sergeev, I. N., Aljutaily, T., Walton, G., \& Huarte, E. (2020). Effects of Synbiotic Supplement on Human Gut Microbiota, Body Composition and Weight Loss in Obesity. Nutrients, 12(1), E222. https://doi.org/10.3390/nu12010222

Torres-Fuentes, C., Schellekens, H., Dinan, T. G., \& Cryan, J. F. (2017). The microbiota-gut-brain axis in obesity. The Lancet. Gastroenterology \& Hepatology, 2(10), 747-756. https://doi.org/10.1016/S2468-1253(17)30147-4 
Vizcaíno, R., Macias-Tomei, C., Márquez S, J. C., Morales, A., \& Torres, N. (2016). USOS CLÍNICOS DE LOS PROBIÓTICOS. Archivos Venezolanos de Puericultura y Pediatría, 79(1), 029-040.

Yang, Y.-J., \& Ni, Y.-H. (2019). Gut microbiota and pediatric obesity/non-alcoholic fatty liver disease. Journal of the Formosan Medical Association = Taiwan $\mathrm{Yi}$ Zhi, 118 Suppl 1, S55-S61. https://doi.org/10.1016/j.jfma.2018.11.006 